\title{
PSEUDO-RIEMANNIAN GEOMETRY IN TERMS OF MULTI-LINEAR BRACKETS
}

\author{
JOAKIM ARNLIND AND GERHARD HUISKEN
}

\begin{abstract}
We show that the pseudo-Riemannian geometry of submanifolds can be formulated in terms of higher order multi-linear maps. In particular, we obtain a Poisson bracket formulation of almost (para-)Kähler geometry.
\end{abstract}

\section{INTRODUCTION}

In a series of papers, the possibility of expressing differential geometry of Riemannian submanifolds as multi bracket algebraic expressions in the function algebra has been investigated [AHH10a, $\mathrm{AHH10b}$, $\mathrm{AHH10c}$, $\mathrm{AHH12}$. More precisely, it was shown that on a $n$-dimensional submanifold $\Sigma$, geometric objects can be written in terms of a $n$-ary alternating multi-linear map acting on the embedding functions. One of the original motivations for studying the problem came from matrix regularizations of surfaces in the context of "Membrane Theory" (cp. Hop82), where smooth functions are mapped to hermitian matrices such that the Poisson bracket of functions correspond to the commutator of matrices (as the matrix dimension becomes large). In this context, matrices corresponding to the embedding coordinates of a surface arise as solutions to equations, which contain matrices associated to surfaces of arbitrary genus. In order to identify the topology of a solution, it is desirable to be able to compute geometric invariants in terms of the embedding matrices and their commutators. This was illustrated in AHH10a where formulas for the discrete scalar curvature and the discrete genus were presented (see also Arn13). Although matrix regularizations provided the original motivation for our work, it is interesting to ask similar questions in the context of general quantizations and non-commutative geometry.

For higher dimensional manifolds, however, one is required to formulate geometry in terms of a $n$-ary bracket, which has no direct analogue as a higher order commutator for operators. This leads to the question if there is perhaps a class of manifolds (of dimension greater than two) for which one may use a Poisson bracket to express geometric quantities. In the following we will demonstrate that almost (para-)Kähler manifolds provide a context where an affirmative answer can be given (cp. AH11 for a preliminary version). In the course of doing so, we shall also consider pseudo-Riemannian manifolds and extend the results of $\mathrm{AHH12}$ to more general types of multi-linear brackets and manifolds of indefinite signature.

\section{Preliminaries}

Let $(M, \eta)$ be a pseudo-Riemannian manifold of dimension $m$, and let $(\Sigma, g)$ be a $n$-dimensional submanifold of $M$ with induced metric $g$. Given local coordinates $x^{1}, \ldots, x^{m}$ on $M$, we consider $\Sigma$ as embedded in $M$ via $x^{i}\left(u^{1}, \ldots, u^{n}\right)$ where 
$u^{1}, \ldots, u^{n}$ are local coordinates on $\Sigma$. Indices $i, j, k, \ldots$ run from 1 to $m$ and indices $a, b, c, \ldots$ run from 1 to $n$. The Levi-Civita connection on $M$ is denoted by $\bar{\nabla}$ (with Christoffel symbols $\bar{\Gamma}_{j k}^{i}$ ) and the Levi-Civita connection on $\Sigma$ by $\nabla$ (with Christoffel symbols $\Gamma_{b c}^{a}$ ). The tangent space $T \Sigma$ is regarded as a subspace of the tangent space $T M$ and at each point of $\Sigma$ one can choose $e_{a}=\left(\partial_{a} x^{i}\right) \partial_{i}$ as basis vectors of $T \Sigma$, and in this basis we define $g_{a b}=\eta\left(e_{a}, e_{b}\right)$.

The formulas of Gauss and Weingarten split the covariant derivative in $M$ into tangential and normal components as

$$
\begin{aligned}
& \bar{\nabla}_{X} Y=\nabla_{X} Y+\alpha(X, Y) \\
& \bar{\nabla}_{X} N=-W_{N}(X)+D_{X} N
\end{aligned}
$$

where $X, Y \in T \Sigma, N \in T \Sigma^{\perp}$ and $\nabla_{X} Y, W_{N}(X) \in T \Sigma$ and $\alpha(X, Y), D_{X} N \in T \Sigma^{\perp}$. It follows that $\alpha(X, Y)=\alpha(Y, X)$ and

$$
\eta(\alpha(X, Y), N)=\eta\left(W_{N}(X), Y\right)
$$

for $N \in T \Sigma^{\perp}$ (Weingarten's equation). From these formulas, one can derive Gauss' equation, which expresses the curvature of the submanifold in terms of the curvature of the ambient manifold and the second fundamental form $\alpha$ :

$$
\begin{aligned}
g(R(X, Y) Z, V)= & \eta(\bar{R}(X, Y) Z, V)-\eta(\alpha(X, Z), \alpha(Y, V)) \\
& +\eta(\alpha(Y, Z), \alpha(X, V)),
\end{aligned}
$$

for $X, Y, Z, V \in T \Sigma$, where $\bar{R}$ and $R$ denote the curvature tensors of $M$ and $\Sigma$ respectively. For more details on submanifolds, please see e.g. KN96a, KN96b.

\section{3. $(N+1)$-BRACKET FORMULATION Of PSEUdo-RIEMANNIAN GEOMETRY}

In the previous section, we introduced $\Sigma$ as a submanifold of $(M, \eta)$, embedded via the coordinates $x^{1}, \ldots, x^{m}$, and equipped with the induced metric $g$. Let us now assume that there exists a $(N+1)$-multilinear map

$$
\{\cdot, \ldots, \cdot\}: \underbrace{C^{\infty}(\Sigma) \times \cdots \times C^{\infty}(\Sigma)}_{N+1} \rightarrow C^{\infty}(\Sigma)
$$

compatible with the usual associative product in the following way 1

$$
\left\{f_{1}, \ldots, f_{k} g, \ldots, f_{N+1}\right\}=f_{k}\left\{f_{1}, \ldots, g, \ldots, f_{N+1}\right\}+g\left\{f_{1}, \ldots, f_{N+1}\right\},
$$

for $k=1, \ldots, N+1$. Furthermore, we introduce multi-indices $I=\left(i_{1} i_{2} \cdots i_{N}\right)$, $\vec{a}=\left(a_{1} \cdots a_{N}\right)$ and set

$$
\begin{aligned}
& \left\{f, \vec{x}^{I}\right\}=\left\{f, x^{i_{1}}, x^{i_{2}}, \ldots, x^{i_{N}}\right\} \\
& \partial_{\vec{a}} \vec{x}^{I}=\left(\partial_{a_{1}} x^{i_{1}}\right)\left(\partial_{a_{2}} x^{i_{2}}\right) \cdots\left(\partial_{a_{N}} x^{i_{N}}\right) \\
& \eta_{I J}=\eta_{i_{1} j_{1}} \eta_{i_{2} j_{2}} \cdots \eta_{i_{N} j_{N}} \\
& g_{\vec{a} \vec{c}}=g_{a_{1} c_{1}} g_{a_{2} c_{2}} \cdots g_{a_{N} c_{N}},
\end{aligned}
$$

as well as

$$
\mathcal{P}^{i I}=\frac{1}{\sqrt{N !}}\left\{x^{i}, \vec{x}^{I}\right\}
$$

\footnotetext{
${ }^{1}$ Note that we have not assumed antisymmetry of the bracket; although our examples are of this kind, it is not necessary to develop the theory.
} 
Since we seek to formulate the metric geometry of $(\Sigma, g)$ with the help of the above bracket, one needs to assume a relation to the metric $g$. Hence, we will in the following assume that there exist $0<\gamma \in C^{\infty}(\Sigma)$ and $\epsilon \in\{-1,1\}$ such that

$$
\mathcal{P}^{i I} \mathcal{P}^{j J} \eta_{I J}=\epsilon \gamma^{2} g^{a b}\left(\partial_{a} x^{i}\right)\left(\partial_{b} x^{j}\right),
$$

where $g^{a b}$ are the components of the inverse of the metric $g$. Introducing a multivector $\theta$ such that

$$
\left\{f, f_{1}, \ldots, f_{N}\right\}=\theta^{a \vec{a}}\left(\partial_{a} f\right)\left(\partial_{a_{1}} f_{1}\right) \cdots\left(\partial_{a_{N}} f_{N}\right)
$$

equation (3.1) can be written as

$$
\epsilon \gamma^{2} g^{a c}=\frac{1}{N !} \theta^{a \vec{a}} \theta^{c \vec{c}} g_{\vec{a} \vec{c}}
$$

(note that this kind of compatibility condition, and the relation to Riemannian geometry has also been studied in the context of matrix models BS10]). If desired, relation (3.1) can be put in a slightly more algebraic form as

$$
\mathcal{P}^{i I} \eta_{I J} \mathcal{P}^{j J} \eta_{j k} \mathcal{P}^{k}\left(f_{1}, \ldots, f_{N}\right)=\epsilon \gamma^{2} \mathcal{P}^{i}\left(f_{1}, \ldots, f_{N}\right)
$$

for all $f_{1}, \ldots, f_{N} \in C^{\infty}(\Sigma)$, where

$$
\mathcal{P}^{i}\left(f_{1}, \ldots, f_{N}\right)=\frac{1}{\sqrt{N !}}\left\{x^{i}, f_{1}, \ldots, f_{N}\right\} .
$$

Although relation (3.1) might look unnatural at first sight, let us point out a number of situations in which it holds true.

Example 3.1 (Pseudo-Riemannian manifolds). Let $(\Sigma, g)$ be a pseudo-Riemannian manifold of dimension $n$ and set

$$
\left\{f_{1}, \ldots, f_{n}\right\}=\frac{1}{\rho} \varepsilon^{a_{1} \cdots a_{n}}\left(\partial_{a_{1}} f_{1}\right) \cdots\left(\partial_{a_{n}} f_{n}\right),
$$

(giving $N+1=n$ ) where $\rho$ is an arbitrary density. Then one computes

$$
\begin{aligned}
\mathcal{P}^{i I} \mathcal{P}^{j J} \eta_{I J}= & \frac{1}{\rho^{2}(n-1) !} \varepsilon^{a a_{1} \cdots a_{n-1}} \varepsilon^{c c_{1} \cdots c_{n-1}}\left(\partial_{a} x^{i}\right)\left(\partial_{c} x^{j}\right)\left(\partial_{a_{1}} x^{i_{1}}\right) \eta_{i_{1} j_{1}}\left(\partial_{c_{1}} x^{j_{1}}\right) \\
& \quad \cdots \times\left(\partial_{a_{n-1}} x^{i_{n-1}}\right) \eta_{i_{n-1} j_{n-1}}\left(\partial_{c_{n-1}} x^{j_{n-1}}\right) \\
= & \frac{1}{\rho^{2}(n-1) !} \varepsilon^{a a_{1} \cdots a_{n-1}} \varepsilon^{c c_{1} \cdots c_{n-1}}\left(\partial_{a} x^{i}\right)\left(\partial_{c} x^{j}\right) g_{a_{1} c_{1}} \cdots g_{a_{n-1} c_{n-1}} \\
= & \frac{g}{\rho^{2}} g^{a c}\left(\partial_{a} x^{i}\right)\left(\partial_{c} x^{j}\right)
\end{aligned}
$$

since the next to last expression is simply the cofactor expansion of the matrix corresponding to the inverse of the metric $g$. Thus, (3.1) is fulfilled with $\epsilon=\operatorname{sgn}(g)$ and $\gamma=\sqrt{|g|} / \rho$, where $g$ denotes the determinant of the metric. In particular, one may use a Poisson bracket to describe the geometry of a 2-dimensional manifold of arbitrary signature. Note that the geometry of pseudo-Riemannian surfaces in terms of Poisson brackets was worked out in Hin11, following the work previously done in $\mathrm{AHH} 10 \mathrm{c}$.

Example 3.2 (Almost Kähler manifolds). Let $(\Sigma, g, J)$ be an almost Kähler manifold with the associated Kähler form

$$
\omega(X, Y)=g(X, J(Y)),
$$


and Poisson bivector $\theta$ as the inverse of $\omega$ (where $J$ denotes the almost complex structure). That is, in this setting one has $N=1$, and

$$
\left\{f_{1}, f_{2}\right\}=\theta^{a b}\left(\partial_{a} f_{1}\right)\left(\partial_{b} f_{2}\right) .
$$

On an almost Kähler manifold it holds that $J^{a}{ }_{b}=-\theta^{a c} g_{c b}$, and $J^{2}=-\mathbb{1}$ gives

$$
g^{a b}=\theta^{a p} \theta^{b q} g_{p q}
$$

which implies that equation (3.2) is satisfied with $\epsilon=\gamma=1$.

Example 3.3 (Indefinite Kähler manifolds). An indefinite (almost) Kähler manifold is an (almost) Kähler manifold where the metric is not necessarily positive definite. Since the complex structure preserves the causal type of vectors (spacelike, timelike or null), any subspace of vectors of a fixed causality is left invariant by the complex structure. Therefore, the index of $g$ (i.e. the dimension of the largest subspace on which $g$ is negative definite) has to be an even integer; that is, the signature of $g$ is of the form $(2 s, 2 n-2 s)$ for $0 \leq s \leq n$. In this case, equation (3.3) still holds, which implies that (3.2) is satisfied with $\epsilon=\gamma=1$, as in the case of ordinary Kähler manifolds. (Please see BR82 for more information on indefinite Kähler manifolds.)

Example 3.4 (Para-Kähler manifolds). An almost para-hermitian manifold $(\Sigma, g, J)$ is a pseudo-Riemannian manifold $(\Sigma, g)$ together with a map $J: T \Sigma \rightarrow T \Sigma$ such that $J^{2}=\mathbb{1}$ and $g(X, Y)=-g(J(X), J(Y))$. If the associated Kähler form $\omega(X, Y)=g(X, J Y)$ is closed, then $(\Sigma, g, J)$ is called an almost para-Kähler manifold. Since $J$ is invertible and maps a vector of negative norm to a vector of positive norm, the signature of $g$ has to be of the form $(n, n)$ (see e.g. [CFG96] for more details). In this case, one derives that the Poisson bivector $\theta$ is given by $\theta^{a b}=J^{a}{ }_{c} g^{c b}$, and $J^{2}=\mathbb{1}$ implies that

$$
g^{a b}=-\theta^{a p} \theta^{b q} g_{p q}
$$

Thus, (3.2) is fulfilled with $\epsilon=-1$ and $\gamma=1$.

Example 3.5. In the above examples, we have considered (para-)Kähler manifolds, which implies that $\gamma=1$. Let us note that manifolds fulfilling (3.2), with $\gamma \neq 1$, are related to (para-)Kähler manifolds by rescaling the metric. Namely, if $(\Sigma, g)$ is a Poisson manifold such that

$$
\epsilon \gamma^{2} g^{a b}=\theta^{a p} \theta^{b q} g_{p q}
$$

then $\Sigma$ is a (para-)Kähler manifold with respect to the rescaled metric $\tilde{g}=\gamma^{-1} g$ and the (para-) complex structure $J^{a}{ }_{b}=\epsilon \gamma^{-1} \theta^{a c} g_{c b}$.

Remark 3.6. Note that (3.2) provides a natural (at least in this context) generalization of Kähler manifolds to $(N+1)$-brackets. Namely, just as (in the case $\gamma=1$ ) (3.3) expresses the fact that $\theta^{a c} g_{c b}$ squares to $-\delta_{b}^{a}$, equation (3.2) tells us that the "square" of the multivector $\theta$ fulfills

$$
\theta^{a \vec{a}} \theta_{b \vec{a}}=\delta_{b}^{a},
$$

giving a relation between $\theta$ and the metric $g$ on $\Sigma$. 
The right hand side of equation (3.1) is more or less the projection operator from $T M$ to $T \Sigma$. Therefore, one introduces

$$
\mathcal{D}^{i j}=\frac{\epsilon}{\gamma^{2}} \mathcal{P}^{i I} \mathcal{P}^{j J} \eta_{I J}=g^{a b}\left(\partial_{a} x^{i}\right)\left(\partial_{b} x^{j}\right),
$$

and sets $\mathcal{D}(X)=\mathcal{D}^{i j} \eta_{j k} X^{k} \partial_{i}$ for $X=X^{i} \partial_{i} \in T M$; moreover, one notes that $\mathcal{D}$ is symmetric, i.e. $\mathcal{D}^{i j}=\mathcal{D}^{j i}$. The factor $\epsilon \gamma^{2}$ is not independent of the bracket, and can be computed from it via

$$
\frac{\epsilon}{n} \mathcal{P}^{i I} \mathcal{P}^{j J} \eta_{I J} \eta_{i j}=\frac{\epsilon^{2} \gamma^{2}}{n} g^{a b} g_{a b}=\gamma^{2} .
$$

Proposition 3.7. The map $\mathcal{D}: T M \rightarrow T M$ is the orthogonal projection onto $T \Sigma$.

Proof. One easily sees that $\mathcal{D}$ is symmetric with respect to $\eta$ (giving an orthogonal projection); namely, one computes

$$
\eta(X, \mathcal{D}(Y))=\eta_{i j} X^{i} \mathcal{D}^{j k} \eta_{k l} Y^{l}=\eta_{k l} \mathcal{D}^{k j} \eta_{j i} X^{i} Y^{l}=\eta(\mathcal{D}(X), Y)
$$

since $\mathcal{D}^{j k}=\mathcal{D}^{k j}$. Moreover, using equation (3.1) one computes that

$$
\begin{aligned}
\left(\mathcal{D}^{2}\right)^{i j} & =\mathcal{D}^{i k} \eta_{k l} \mathcal{D}^{l j}=g^{a b}\left(\partial_{a} x^{i}\right)\left(\partial_{b} x^{k}\right) \eta_{k l} g^{p q}\left(\partial_{p} x^{l}\right)\left(\partial_{q} x^{j}\right) \\
& =g^{a b} g^{p q} g_{b p}\left(\partial_{a} x^{i}\right)\left(\partial_{q} x^{j}\right)=g^{a q}\left(\partial_{a} x^{i}\right)\left(\partial_{q} x^{j}\right)=\mathcal{D}^{i j},
\end{aligned}
$$

which shows that $\mathcal{D}$ is indeed projection operator. Now, let us choose $X \in T \Sigma$ and write $X=X^{a}\left(\partial_{a} x^{i}\right) \partial_{i}$. One then computes

$$
\mathcal{D}(X)=g^{a b}\left(\partial_{a} x^{i}\right)\left(\partial_{b} x^{j}\right) \eta_{j k} X^{c}\left(\partial_{c} x^{k}\right) \partial_{i}=g^{a b} g_{b c}\left(\partial_{a} x^{i}\right) X^{c} \partial_{i}=X,
$$

showing that $\mathcal{D}$ is indeed the projection onto $T \Sigma$.

It is also convenient to introduce the projection $\Pi$ onto the complementary space $T \Sigma^{\perp}$; i.e we set $\Pi=\mathbb{1}-\mathcal{D}$. Having the projection at hand, one immediately obtains the Levi-Civita connection of $\Sigma$ as

$$
\nabla_{X} Y^{i}=\mathcal{D}\left(\bar{\nabla}_{X} Y\right)^{i}=\mathcal{D}^{i}{ }_{j}\left(X^{k} \partial_{k}\left(Y^{j}\right)+\bar{\Gamma}_{k l}^{j} X^{k} Y^{l}\right)
$$

for $X, Y \in T \Sigma$. However, the above formula has an explicit derivative appearing in it, and can not be completely written in terms of $(N+1)$-brackets. Therefore, it is convenient to introduce

$$
\hat{\nabla}_{X} Y=\bar{\nabla}_{\mathcal{D}(X)} Y,
$$

for which it holds that $\hat{\nabla}_{X} Y=\bar{\nabla}_{X} Y$, whenever $X \in T \Sigma$, and

$$
\hat{\nabla}_{j} Y^{i}=\mathcal{D}_{j}\left(Y^{i}\right)+\mathcal{D}_{j}{ }^{l} \bar{\Gamma}_{l k}^{i} Y^{k}
$$

with

$$
\mathcal{D}^{i}(f)=\mathcal{D}^{i j}\left(\partial_{j} f\right)=\frac{\epsilon}{\gamma^{2} N !}\left\{x^{i}, \vec{x}^{I}\right\}\left\{f, \vec{x}^{J}\right\} \eta_{I J} .
$$

Note that equation (3.5) is written entirely in terms of $(N+1)$-brackets:

$$
\hat{\nabla}^{j} Y^{i}=\frac{\epsilon}{\gamma^{2} N !}\left\{Y^{i}, \vec{x}^{I}\right\}\left\{x^{j}, \vec{x}^{J}\right\} \eta_{I J}+\frac{\epsilon}{\gamma^{2} N !}\left\{x^{j}, \vec{x}^{I}\right\}\left\{x^{l}, \vec{x}^{J}\right\} \eta_{I J} \bar{\Gamma}_{l k}^{i} Y^{k}
$$

Thus, the Levi-Civita connection on $\Sigma$ may also be written in terms of $(N+1)$ brackets as

$$
\nabla_{j} Y^{i}=\mathcal{D}^{i}{ }_{k} \hat{\nabla}_{j} Y^{k}
$$


Let us proceed to show that $\hat{\nabla}$ is (not surprisingly) closely related to the curvature of $(M, \eta)$. To start with, let us collect a few computations related to the second fundamental form in the following lemma:

Lemma 3.8. For $X, Y, Z, V \in T \Sigma$ it holds that

$$
\begin{aligned}
& \alpha(X, Y)^{i}=-\left(\hat{\nabla}_{j} \Pi_{k}^{i}\right) X^{j} Y^{k} \\
& \eta(\alpha(X, Y), \alpha(Z, V))=\left(\hat{\nabla}_{i} \Pi_{m j}\right)\left(\hat{\nabla}_{k} \Pi_{l}^{m}\right) X^{i} Y^{j} Z^{k} V^{l} .
\end{aligned}
$$

In particular, since $\alpha(X, Y)=\alpha(Y, X)$ it follows from (3.7) that

$$
X^{i} Y^{j}\left(\hat{\nabla}_{i} \mathcal{D}_{j k}-\hat{\nabla}_{j} \mathcal{D}_{i k}\right)=0 .
$$

Proof. For $X, Y \in T \Sigma$, the second fundamental form is given by

$$
\alpha(X, Y)^{i}=\Pi\left(\bar{\nabla}_{X} Y\right)^{i}=\Pi\left(\hat{\nabla}_{X} Y\right)^{i}=\Pi_{k}^{i} X^{l} \hat{\nabla}_{l} Y^{k}=-X^{l} Y^{k} \hat{\nabla}_{l} \Pi^{i}{ }_{k}
$$

since $\Pi(Y)=0$. The second formula follows immediately from this result. The last formula is proved by inserting $\Pi=\mathbb{1}-\mathcal{D}$ into equation (3.7) and using that $\alpha(X, Y)=\alpha(Y, X)$.

The next results confirms that the commutator of $\hat{\nabla}_{i}$ and $\hat{\nabla}_{i}$ does indeed give the curvature of $(M, \eta)$ :

Proposition 3.9. Let $\bar{R}$ be the curvature tensor of $(M, \eta)$. For $X, Y \in T \Sigma$ and $U \in T M$ it holds that

$$
X^{i} Y^{j}\left(\hat{\nabla}_{i} \hat{\nabla}_{j} U^{k}-\hat{\nabla}_{j} \hat{\nabla}_{i} U^{k}\right)=\bar{R}(X, Y) U^{k}
$$

Proof. From the definition of $\hat{\nabla}$ one obtains

$$
\begin{aligned}
X^{i} Y^{j} & \left(\hat{\nabla}_{i} \hat{\nabla}_{j} U^{k}-\hat{\nabla}_{j} \hat{\nabla}_{i} U^{k}\right)=X^{i} Y^{j}\left(\mathcal{D}_{i}{ }^{l} \bar{\nabla}_{l}\left(\mathcal{D}_{j}{ }^{m} \bar{\nabla}_{m} U^{k}\right)-\mathcal{D}_{j}{ }^{l} \bar{\nabla}_{l}\left(\mathcal{D}_{i}{ }^{m} \bar{\nabla}_{m} U^{k}\right)\right) \\
& =X^{i} Y^{j}\left(\left[\bar{\nabla}_{i}, \bar{\nabla}_{j}\right] U^{k}+\hat{\nabla}_{i}\left(\mathcal{D}_{j}{ }^{m}\right) \bar{\nabla}_{m} U^{k}-\hat{\nabla}_{j}\left(\mathcal{D}_{i}{ }^{m}\right) \bar{\nabla}_{m} U^{k}\right) \\
& =X^{i} Y^{j}\left[\bar{\nabla}_{i}, \bar{\nabla}_{j}\right] U^{k}=\bar{R}(X, Y) U^{k}
\end{aligned}
$$

by using equation (3.9) in Lemma 3.8

By using Gauss' equation (2.3) and Lemma 3.8, we proceed to show that the curvature of $(\Sigma, g)$ can be expressed in terms of $\hat{\nabla}$ and the projection $\Pi$.

Proposition 3.10. Let $\bar{R}$ and $R$ be the curvature tensors of $(M, \eta)$ and $(\Sigma, g)$ respectively. For $X, Y, Z, V \in T \Sigma$ it holds that

$$
R(X, Y, Z, V)=\left(\bar{R}_{i j k l}+\left(\hat{\nabla}_{k} \Pi_{m i}\right)\left(\hat{\nabla}_{l} \Pi_{j}^{m}\right)-\left(\hat{\nabla}_{k} \Pi_{m j}\right)\left(\hat{\nabla}_{l} \Pi^{m}{ }_{i}\right)\right) X^{i} Y^{j} Z^{k} V^{l} .
$$

Proof. The formula for the curvature of $(\Sigma, g)$ is obtained by inserting the expression for the second fundamental form, found in equation (3.7) in Lemma 3.8, into Gauss' equation (2.3).

To derive formulas for the scalar and Ricci curvatures one notes that the trace of $T: T \Sigma \times T \Sigma \rightarrow C^{\infty}(\Sigma)$ may be computed as

$$
\operatorname{Tr} T=g^{a b} T\left(e_{a}, e_{b}\right)=g^{a b} T_{i j}\left(e_{a}\right)^{i}\left(e_{b}\right)^{j}=g^{a b}\left(\partial_{a} x^{i}\right)\left(\partial_{b} x^{j}\right) T_{i j}=\mathcal{D}^{i j} T_{i j} .
$$

Thus, when computing the trace over $T \Sigma$, one may effectively use $\mathcal{D}^{i j}$ instead of $\eta^{i j}$ (which corresponds to the trace in $T M$ ). In this way, one immediately obtains formulas for the scalar and Ricci curvatures: 
Proposition 3.11. Let $\bar{R}$ denote the curvature tensor of $(M, \eta)$, and let Ric and $S$ denote the Ricci and scalar curvatures of $(\Sigma, g)$, respectively. For $X, Y \in T \Sigma$ it holds that

$$
\begin{aligned}
\operatorname{Ric}(X, Y) & =\left(\mathcal{D}^{k l} \bar{R}_{k i l j}+\left(\hat{\nabla}_{k} \Pi^{k l}\right)\left(\hat{\nabla}_{j} \Pi_{i l}\right)-\left(\hat{\nabla}_{k} \Pi_{l i}\right)\left(\hat{\nabla}_{j} \Pi^{l k}\right)\right) X^{i} Y^{j} \\
S & =\mathcal{D}^{i j} \mathcal{D}^{k l} \bar{R}_{k i l j}+\left(\hat{\nabla}^{k} \Pi_{k l}\right)\left(\hat{\nabla}_{i} \Pi^{i l}\right)-\left(\hat{\nabla}_{k} \Pi_{i l}\right)\left(\hat{\nabla}^{i} \Pi^{k l}\right) .
\end{aligned}
$$

Gauss' equation relates the curvature in the tangential direction (i.e. along the submanifold) to the curvature of the ambient space. Let us now study curvature in the normal directions; for this reason, we introduce

$$
\mathcal{B}_{N}^{i j}=-\hat{\nabla}^{i} N^{j}=-\frac{\epsilon}{\gamma^{2} N !}\left\{x^{i}, \vec{x}^{I}\right\}\left\{N^{j}, \vec{x}^{J}\right\} \eta_{I J}-\frac{\epsilon}{\gamma^{2} N !}\left\{x^{i}, \vec{x}^{I}\right\}\left\{x^{k}, \vec{x}^{J}\right\} \eta_{I J} \bar{\Gamma}_{k l}^{j} N^{l},
$$

for any $N=N^{i} \partial_{i} \in T \Sigma^{\perp}$, and note that this is again an expression in terms of $(N+1)$-brackets. From this definition we induce a mapping $\mathcal{B}_{N}: T M \rightarrow T M$ by setting $\mathcal{B}_{N}(X)=\mathcal{B}_{N}^{i j} \eta_{j k} X^{k} \partial_{i}$. It is clear from the definition that $\mathcal{B}_{N}(X) \in T \Sigma$ for any $X \in T M$ and $N \in T \Sigma^{\perp}$. Furthermore, it turns out that $\mathcal{B}_{N}$ captures the tangential and normal components of $\bar{\nabla}_{X} N$ in the following way:

Proposition 3.12. For $X \in T \Sigma$ and $N, \tilde{N} \in T \Sigma^{\perp}$ it holds that

$$
\begin{aligned}
& \mathcal{B}_{N}(X)=W_{N}(X) \\
& \eta\left(\mathcal{B}_{N}(\tilde{N}), X\right)=-\eta\left(D_{X} N, \tilde{N}\right),
\end{aligned}
$$

where $W_{N}(X)$ and $D_{X} N$ denote the tangential and normal components of $\bar{\nabla}_{X} N$ respectively (cp. equation (2.2)).

Proof. Let us first note that, due to the symmetry of $\alpha$, it holds that

$$
\begin{aligned}
& \eta(\alpha(X, Y), N)=\eta(\alpha(Y, X), N) \Leftrightarrow \eta\left(W_{N}(X), Y\right)=\eta\left(W_{N}(Y), X\right) \\
& \quad \Leftrightarrow \quad \eta\left(\bar{\nabla}_{X} N, Y\right)=\eta\left(\bar{\nabla}_{Y} N, X\right),
\end{aligned}
$$

for $X, Y \in T \Sigma$ and $N \in T \Sigma^{\perp}$. Using this, one computes that

$$
\begin{aligned}
\mathcal{B}_{N}(X)^{i} & =-\mathcal{D}^{i k}\left(\bar{\nabla}_{k} N^{j}\right) X_{j}=-g^{a b}\left(\partial_{a} x^{i}\right)\left(\partial_{b} x^{k}\right)\left(\bar{\nabla}_{k} N^{j}\right)\left(\partial_{c} x^{l}\right) \eta_{j l} X^{c} \\
& =-g^{a b}\left(\partial_{a} x^{i}\right) \eta\left(\bar{\nabla}_{e_{b}} N, e_{c}\right) X^{c}=-g^{a b}\left(\partial_{a} x^{i}\right) \eta\left(\bar{\nabla}_{e_{c}} N, e_{b}\right) X^{c} \\
& =-g^{a b}\left(\partial_{a} x^{i}\right) \eta\left(\bar{\nabla}_{X} N, e_{b}\right)=-\mathcal{D}\left(\bar{\nabla}_{X} N\right)^{i}=W_{N}(X)^{i} .
\end{aligned}
$$

Moreover, for $N, \tilde{N} \in T \Sigma^{\perp}$ and $X \in T \Sigma$ one obtains

$$
\eta\left(\mathcal{B}_{N}(\tilde{N}), X\right)=-X_{i}\left(\hat{\nabla}^{i} N^{j}\right) \tilde{N}_{j}=-\eta\left(\bar{\nabla}_{X} N, \tilde{N}\right)=-\eta\left(D_{X} N, \tilde{N}\right),
$$

which proves formula (3.11).

Note that since $\eta\left(W_{N}(X), Y\right)=\eta(\alpha(X, Y), N)$ (Weingarten's equation), it follows from Proposition 3.12 that

$$
\eta(\alpha(X, Y), N)=\eta\left(\mathcal{B}_{N}(X), Y\right) .
$$

As a complement to Gauss' equation, the Codazzi-Mainardi equations express the normal component of the curvature in the ambient space. Due to the symmetries of the curvature tensor, one can immediately write

$$
\eta(\bar{R}(X, Y) Z, N)=-\eta(\bar{R}(X, Y) N, Z)=-X^{i} Y^{j} Z^{l}\left(\hat{\nabla}_{j} \hat{\nabla}_{i} N_{l}-\hat{\nabla}_{i} \hat{\nabla}_{j} N_{l}\right),
$$


and from the definition of $\mathcal{B}_{N}^{i j}$ one obtains

$$
\begin{aligned}
\eta(\bar{R}(X, Y) Z, N) & =X_{i} Y_{j} Z_{l}\left(\hat{\nabla}^{j} \mathcal{B}_{N}^{i l}-\nabla^{i} \mathcal{B}_{N}^{j l}\right) \\
& =\eta\left(\left(\hat{\nabla}_{Y} \mathcal{B}_{N}\right)(Z), X\right)-\eta\left(\left(\hat{\nabla}_{X} \mathcal{B}_{N}\right)(Z), Y\right) .
\end{aligned}
$$

Let us point out that this way of writing the normal component of the curvature has a close resemblance to an expression in terms of a connection in $T \Sigma \oplus T \Sigma^{\perp}$, defined by combining $D_{X}$ and $\nabla_{X}$. Namely, by writing

$$
\left(\widetilde{\nabla}_{X} \alpha\right)(Y, Z)=D_{X} \alpha(Y, Z)-\alpha\left(\nabla_{X} Y, Z\right)-\alpha\left(Y, \nabla_{X} Z\right)
$$

the normal component of the curvature is $\left(\widetilde{\nabla}_{X} \alpha\right)(Y, Z)-\left(\widetilde{\nabla}_{Y} \alpha\right)(X, Z)$ (see KN96b, page 25$)$. Let us verify directly that they are, in fact, the same. One computes

$$
\begin{aligned}
& \eta\left(\left(\hat{\nabla}_{X} \mathcal{B}_{N}\right)(Z), Y\right)=\eta\left(\left(\bar{\nabla}_{X} \mathcal{B}_{N}\right)(Z), Y\right)=\eta\left(\bar{\nabla}_{X} \mathcal{B}_{N}(Z), Y\right)-\eta\left(\mathcal{B}_{N}\left(\bar{\nabla}_{X} Z\right), Y\right) \\
& \quad=\eta\left(\nabla_{X} \mathcal{B}_{N}(Z), Y\right)-\eta\left(\mathcal{B}_{N}\left(\nabla_{X} Z\right), Y\right)-\eta\left(\mathcal{B}_{N} \alpha(X, Z), Y\right) \\
& \quad=X \cdot \eta\left(\mathcal{B}_{N}(Z), Y\right)-\eta\left(\mathcal{B}_{N}(Z), \nabla_{X} Y\right)-\eta\left(\mathcal{B}_{N}\left(\nabla_{X} Z\right), Y\right)-\eta\left(\mathcal{B}_{N} \alpha(X, Z), Y\right),
\end{aligned}
$$

and using Proposition 3.12 together with equation (3.12) one gets

$$
\begin{gathered}
\eta\left(\left(\hat{\nabla}_{X} \mathcal{B}_{N}\right)(Z), Y\right)=X \cdot \eta(\alpha(Y, Z), N)-\eta\left(\alpha\left(\nabla_{X} Y, Z\right), N\right) \\
-\eta\left(\alpha\left(Y, \nabla_{X} Z\right), N\right)+\eta\left(D_{Y} N, \alpha(X, Z)\right) \\
=X \cdot \eta(\alpha(Y, Z), N)+Y \cdot \eta(\alpha(X, Z), N)-\eta\left(D_{Y} \alpha(X, Z), N\right) \\
-\eta\left(\alpha\left(\nabla_{X} Y, Z\right), N\right)-\eta\left(\alpha\left(Y, \nabla_{X} Z\right), N\right),
\end{gathered}
$$

which implies that

$$
\eta\left(\left(\hat{\nabla}_{Y} \mathcal{B}_{N}\right)(Z), X\right)-\eta\left(\left(\hat{\nabla}_{X} \mathcal{B}_{N}\right)(Z), Y\right)=\eta\left(\left(\widetilde{\nabla}_{X} \alpha\right)(Y, Z)-\left(\widetilde{\nabla}_{Y} \alpha\right)(X, Z), N\right) .
$$

\section{The Laplace operator}

The gradient of a function $f \in C^{\infty}(\Sigma)$ may be written as

$$
\mathcal{D}^{i}(f) \partial_{i}=g^{a b}\left(\partial_{a} f\right)\left(\partial_{b} x^{i}\right) \partial_{i}=g^{a b}\left(\partial_{a} f\right) e_{b}=\nabla f,
$$

and the divergence of an element $X \in T \Sigma$ as

$$
\begin{aligned}
\hat{\nabla}_{i} X^{i} & =\mathcal{D}^{k}{ }_{i} \bar{\nabla}_{k} X^{i}=g^{a b}\left(\partial_{a} x^{k}\right)\left(\partial_{b} x^{l}\right) \eta_{l i} \bar{\nabla}_{k} X^{i}=g^{a b}\left(\partial_{b} x^{l}\right) \eta_{l i}\left(\bar{\nabla}_{e_{a}} X\right)^{i} \\
& =g^{a b}\left(\partial_{b} x^{l}\right) \eta_{l i}\left(\nabla_{e_{a}} X+\alpha\left(e_{a}, X\right)\right)^{i}=g^{a b}\left(\partial_{b} x^{l}\right) \eta_{l i}\left(\nabla_{e_{a}} X\right)^{i} \\
& =g^{a b}\left(\partial_{b} x^{l}\right) \eta_{l i}\left(\nabla_{a} X\right)^{c}\left(\partial_{c} x^{i}\right)=g^{a b} g_{b c} \nabla_{a} X^{c}=\nabla_{a} X^{a}=\operatorname{div}(X) .
\end{aligned}
$$

Consequently, the Laplace-Beltrami operator on $(\Sigma, g)$ may be computed as

$$
\Delta(f)=\operatorname{div}(\operatorname{grad}(f))=\hat{\nabla}_{i} \hat{\nabla}^{i}(f),
$$

where $\hat{\nabla}^{i}(f)=\mathcal{D}^{i}(f)$. Let us now show that one may derive a simpler form of the Laplace operator in certain special cases. Namely, let us first assume that the multivector $\theta$ (defining the $(N+1)$-bracket) is completely antisymmetric, which implies that

$$
\begin{aligned}
& \left\{\gamma^{M}\left\{f, \vec{x}^{I}\right\} \eta_{I J}, \vec{x}^{J}\right\}=\frac{1}{N !} \theta^{a \vec{a}} \partial_{a}\left(\gamma^{M} \theta^{c \vec{c}}\left(\partial_{c} f\right)\left(\partial_{\vec{c}} \vec{x}^{I}\right) \eta_{I J}\right)\left(\partial_{\vec{a}} \vec{x}^{J}\right) \\
& \quad=\frac{1}{N !} \theta^{a \vec{a}} \partial_{a}\left(\gamma^{M} \theta^{c \vec{c}}\left(\partial_{c} f\right)\left(\partial_{\vec{c}} \vec{x}^{I}\right)\left(\partial_{\vec{a}} \vec{x}^{J}\right) \eta_{I J}\right)=\frac{1}{N !} \theta^{a \vec{a}} \partial_{a}\left(\gamma^{M} \theta^{c \vec{c}}\left(\partial_{c} f\right) g_{\vec{a} \vec{c}}\right),
\end{aligned}
$$


since $\theta^{a \vec{a}} \partial_{a}\left(\partial_{\vec{a}} \vec{x}^{J}\right)=0$ due to the antisymmetry of $\theta$. Next, assuming that there exists a function $\rho \in C^{\infty}(\Sigma)$ such that $\partial_{a}\left(\rho \theta^{a \vec{a}}\right)=0$ one obtains

$$
\left\{\gamma^{M}\left\{f, \vec{x}^{I}\right\} \eta_{I J}, \vec{x}^{J}\right\}=\frac{1}{\rho N !} \partial_{a}\left(\rho \gamma^{M} \theta^{a \vec{a}} \theta^{c \vec{c}} g_{\vec{a} \vec{c}} \partial_{c} f\right)=\frac{\epsilon}{\rho} \partial_{a}\left(\rho \gamma^{M+2} g^{a c} \partial_{c} f\right),
$$

by using (3.2). In the case of Example 3.1. $\theta^{a \vec{a}}=\rho^{-1} \varepsilon^{a \vec{a}}$, and it follows immediately that $\theta$ is completely antisymmetric and that $\partial_{a}\left(\rho \theta^{a \vec{a}}\right)=0$. Therefore, one gets

$$
\left\{\gamma^{M}\left\{f, \vec{x}^{I}\right\} \eta_{I J}, \vec{x}^{J}\right\}=\frac{\epsilon}{\rho} \partial_{a}\left(\rho \gamma^{M+2} g^{a c} \partial_{c} f\right)=\frac{\epsilon}{\rho} \partial_{a}\left(\rho^{-M-1} \sqrt{|g|}^{M+2} g^{a c} \partial_{c} f\right)
$$

since $\gamma=\sqrt{|g|} / \rho$, and choosing $M=-1$ gives

$$
\Delta(f)=\frac{\varepsilon}{\gamma}\left\{\gamma^{-1}\left\{f, \vec{x}^{I}\right\} \eta_{I J}, \vec{x}^{J}\right\} .
$$

In Examples 3.23 .4 the $(N+1)$-bracket is a Poisson structure, and by setting $\rho=(\sqrt{\operatorname{det} \theta})^{-1}$ it follows from the Jacobi identity that $\partial_{a}\left(\rho \theta^{a b}\right)=0$; namely, by multiplying the Jacobi identity by $\omega$, the inverse of $\theta$ (i.e. $\omega_{a b} \theta^{b c}=\delta_{a}^{c}$ ), one obtains

$$
\begin{aligned}
& \omega_{a b}\left(\theta^{a p} \partial_{p} \theta^{b c}+\theta^{b p} \partial_{p} \theta^{c a}+\theta^{c p} \partial_{p} \theta^{a b}\right)=0 \Leftrightarrow \\
& \partial_{a} \theta^{a c}=\frac{1}{2} \omega_{a b}\left(\partial_{p} \theta^{b a}\right) \theta^{p c}=\frac{1}{\operatorname{det} \theta}\left(\partial_{p} \operatorname{det} \theta\right) \theta^{p c},
\end{aligned}
$$

since $\partial_{p} \operatorname{det} \theta=(\operatorname{det} \theta) \omega_{a b} \partial_{p} \theta^{b a}$, from which it follows that $\partial_{a}\left(\sqrt{\operatorname{det} \theta}^{-1} \theta^{a c}\right)=0$ (cp. also BS10). These considerations imply that

$$
\left\{\gamma^{M}\left\{f, \vec{x}^{I}\right\} \eta_{I J}, \vec{x}^{J}\right\}=\epsilon \sqrt{\theta} \partial_{a}\left(\sqrt{\operatorname{det} \theta}^{-1} \gamma^{M+2} g^{a c} \partial_{c} f\right) .
$$

Now, the determinant of the relation $\varepsilon \gamma^{2} g^{a b}=\theta^{a p} \theta^{b q} g_{p q}$, together with $\operatorname{dim} \Sigma$ being even, implies that

$$
\sqrt{\operatorname{det} \theta}=\frac{\sqrt{\gamma^{n}}}{\sqrt{|g|}}
$$

Thus, by choosing $M=(n-4) / 2$, the Laplace operator may be written as

$$
\Delta(f)=\frac{\epsilon}{\sqrt{\gamma^{n}}}\left\{\sqrt{\gamma^{n-4}}\left\{f, \vec{x}^{I}\right\} \eta_{I J}, \vec{x}^{J}\right\},
$$

for almost (para-)Kähler manifolds.

\section{Explicit formulas when $(M, \eta)=\left(\mathbb{R}^{m}, \delta\right)$}

In the course of rewriting geometry in terms of $(N+1)$-brackets, we have developed a notation which makes the expressions for most quantities rather short and concise. Of course, in the general case, writing out all the brackets and Christoffel symbols will produce formulas that are quite lengthy. However, in the particular situation when the ambient space is $\mathbb{R}^{m}$ equipped with the metric $g_{a b}=\delta_{a b}$ explicit expressions are considerably reduced in size. Apart from being simple, it is also an interesting case since it is generic, in the sense that any manifold can be isometrically embedded in some Euclidean space $\left(\mathbb{R}^{m}, \delta\right)$ ( $[$ Nas56]), and many manifolds do have a concrete presentation in such a way. Let us therefore, in this case, present explicit formulas for some of the geometric objects for which we have developed an $(N+1)$-bracket formulation. Note that for pseudo-Riemannian manifolds a similar statement holds where one may always isometrically embed a pseudo-Riemannian 
manifold into pseudo-Euclidean space (see [Cla70 for details). The formulas given below can easily be extended to this setting.

When $(M, \eta)=\left(\mathbb{R}^{m}, \delta\right)$ there is no difference between upper and lower indices; therefore, we shall leave all (multi)-indices in the upper position and assume that all repeated (multi-)indices are summed over from 1 to $m$. Moreover, since $\left(\mathbb{R}^{m}, \delta\right)$ is flat, it holds that $\hat{\nabla}^{i} X^{j}=\mathcal{D}^{i}\left(X^{j}\right)$. The factor $\gamma^{2}$ will be eliminated via

$$
\gamma^{2}=\frac{\epsilon}{n} \mathcal{P}^{i I} \mathcal{P}^{i I}=\frac{\epsilon}{n N !}\left\{x^{i}, \vec{x}^{I}\right\}\left\{x^{i}, \vec{x}^{I}\right\},
$$

giving, for instance,

$$
\mathcal{D}^{i k}=\frac{\epsilon}{\gamma^{2} N !}\left\{x^{i}, \vec{x}^{I}\right\}\left\{x^{k}, \vec{x}^{I}\right\}=n \frac{\left\{x^{i}, \vec{x}^{I}\right\}\left\{x^{k}, \vec{x}^{I}\right\}}{\left\{x^{j}, \vec{x}^{J}\right\}\left\{x^{j}, \vec{x}^{J}\right\}} .
$$

In the same way, one derives the following expressions:

$$
\begin{aligned}
\nabla^{i}(f) & =n \frac{\left\{f, \vec{x}^{I}\right\}\left\{x^{i}, \vec{x}^{I}\right\}}{\left\{x^{j}, \vec{x}^{J}\right\}\left\{x^{j}, \vec{x}^{J}\right\}} \quad \operatorname{div}(X)=n \frac{\left\{X^{i}, \vec{x}^{I}\right\}\left\{x^{i}, \vec{x}^{I}\right\}}{\left\{x^{j}, \vec{x}^{J}\right\}\left\{x^{j}, \vec{x}^{J}\right\}} \\
W_{N}(X)^{i} & =-n \frac{\left\{N^{j}, \vec{x}^{i}\right\}\left\{x^{i}, \vec{x}^{I}\right\}}{\left\{x^{j}, \vec{x}^{J}\right\}\left\{x^{j}, \vec{x}^{J}\right\}} X^{j} \quad \Delta(f)=n^{2} \frac{\left\{\frac{\left\{f, \vec{x}^{L}\right\}\left\{x^{i}, \vec{x}^{L}\right\}}{\left\{x^{k}, \vec{x}^{K}\right\}\left\{x^{k}, \vec{x}^{K}\right\}}, \vec{x}^{I}\right\}\left\{x^{i}, \vec{x}^{I}\right\}}{\left\{x^{j}, \vec{x}^{J}\right\}\left\{x^{j}, \vec{x}^{J}\right\}} \\
\nabla_{X} Y^{i} & =n^{2} \frac{X^{k}\left\{x^{k}, \vec{x}^{J}\right\}\left\{Y^{j}, \vec{x}^{J}\right\}\left\{x^{j}, \vec{x}^{I}\right\}\left\{x^{i}, \vec{x}^{I}\right\}}{\left(\left\{x^{l}, \vec{x}^{L}\right\}\left\{x^{l}, \vec{x}^{L}\right\}\right)^{2}} .
\end{aligned}
$$

The scalar curvature may be written as

$$
\begin{aligned}
S=n^{4} & \frac{\left\{x^{k}, \vec{x}^{I}\right\}\left\{\frac{\left\{x^{k}, \vec{x}^{K}\right\}\left\{x^{l}, \vec{x}^{K}\right\}}{\left\{x^{j}, \vec{x}^{L}\right\}\left\{x^{j}, \vec{x}^{L}\right\}}, \vec{x}^{I}\right\}\left\{x^{i}, \vec{x}^{J}\right\}\left\{\frac{\left\{x^{i}, \vec{x}^{A}\right\}\left\{x^{l}, \vec{x}^{A}\right\}}{\left\{x^{j^{\prime}}, \vec{x}^{B}\right\}\left\{x^{j^{\prime}}, \vec{x}^{B}\right\}}, \vec{x}^{J}\right\}}{\left(\left\{x^{m}, \vec{x}^{M}\right\}\left\{x^{m}, \vec{x}^{M}\right\}\right)^{2}} \\
& -n^{4} \frac{\left\{x^{k}, \vec{x}^{I}\right\}\left\{\frac{\left\{x^{i}, \vec{x}^{K}\right\}\left\{x^{l}, \vec{x}^{K}\right\}}{\left\{x^{j}, \vec{x}^{L}\right\}\left\{x^{j}, \vec{x}^{L}\right\}}, \vec{x}^{I}\right\}\left\{x^{i}, \vec{x}^{J}\right\}\left\{\frac{\left\{x^{k}, \vec{x}^{A}\right\}\left\{x^{l}, \vec{x}^{A}\right\}}{\left\{x^{j^{\prime}}, \vec{x}^{B}\right\}\left\{x^{j^{\prime}}, \vec{x}^{B}\right\}}, \vec{x}^{J}\right\}}{\left(\left\{x^{m}, \vec{x}^{M}\right\}\left\{x^{m}, \vec{x}^{M}\right\}\right)^{2}},
\end{aligned}
$$

and the Codazzi-Mainardi equations become

$$
\left(\left\{\frac{\left\{N^{k}, \vec{x}^{K}\right\}\left\{x^{j}, \vec{x}^{K}\right\}}{\left\{x^{l}, \vec{x}^{L}\right\}\left\{x^{l}, \vec{x}^{L}\right\}}, \vec{x}^{I}\right\}\left\{x^{i}, \vec{x}^{I}\right\}-\left\{\frac{\left\{N^{k}, \vec{x}^{K}\right\}\left\{x^{i}, \vec{x}^{K}\right\}}{\left\{x^{l}, \vec{x}^{L}\right\}\left\{x^{l}, \vec{x}^{L}\right\}}, \vec{x}^{I}\right\}\left\{x^{j}, \vec{x}^{I}\right\}\right) X^{i} Y^{j} Z^{k}=0,
$$

for all $X, Y, Z \in T \Sigma$ and $N \in T \Sigma^{\perp}$. For almost (para-)Kähler manifolds, in which case $\gamma=1$ and $N=1$, the formulas are even more compelling:

$$
\begin{array}{rlrl}
\nabla^{i}(f) & =\epsilon\left\{f, x^{j}\right\}\left\{x^{i}, x^{j}\right\} & \operatorname{div}(X)=\epsilon\left\{X^{i}, x^{j}\right\}\left\{x^{i}, x^{j}\right\} \\
W_{N}(X)^{i} & =-\epsilon\left\{N^{j}, x^{k}\right\}\left\{x^{i}, x^{k}\right\} X^{j} & \Delta(f)=\left\{\left\{f, x^{k}\right\}\left\{x^{i}, x^{k}\right\}, x^{j}\right\}\left\{x^{i}, x^{j}\right\} \\
\nabla_{X} Y^{i} & =X^{k}\left\{x^{k}, x^{l}\right\}\left\{Y^{j}, x^{l}\right\}\left\{x^{j}, x^{m}\right\}\left\{x^{i}, x^{m}\right\},
\end{array}
$$

and the scalar curvature becomes

$$
\begin{aligned}
S= & \left\{x^{k}, x^{i^{\prime}}\right\}\left\{\left\{x^{k}, x^{j}\right\}\left\{x^{l}, x^{j}\right\}, x^{i^{\prime}}\right\}\left\{x^{i}, x^{l^{\prime}}\right\}\left\{\left\{x^{i}, x^{k^{\prime}}\right\}\left\{x^{l}, x^{k^{\prime}}\right\}, x^{l^{\prime}}\right\} \\
& -\left\{x^{k}, x^{i^{\prime}}\right\}\left\{\left\{x^{i}, x^{j}\right\}\left\{x^{l}, x^{j}\right\}, x^{i^{\prime}}\right\}\left\{x^{i}, x^{l^{\prime}}\right\}\left\{\left\{x^{k}, x^{k^{\prime}}\right\}\left\{x^{l}, x^{k^{\prime}}\right\}, x^{l^{\prime}}\right\} .
\end{aligned}
$$

Let us end this section by noting that, when $N=1$, the Codazzi-Mainardi equations are actually Poisson algebraic identities once we assume that equation (3.1) holds. Namely, multiplying (3.1) by $\mathcal{P}^{j k}$ gives $\mathcal{D}^{i j} \mathcal{P}^{j k}=\mathcal{P}^{i k}$ from which it follows that 
$\mathcal{D}^{i j} \mathcal{P}^{j}(f)=\mathcal{P}^{i}(f)$ and $\mathcal{P}^{i j} \mathcal{D}^{j}(f)=\mathcal{P}^{i}(f)$ where $\mathcal{P}^{i}(f)=\left\{x^{i}, f\right\}$. Then one readily proves the following:

Lemma 5.1. If $\mathcal{D}^{i j} \mathcal{P}^{j k}=\mathcal{P}^{i k}$ then it holds that

$$
\left[\mathcal{D}^{i}, \mathcal{D}^{j}\right](f) \mathcal{P}^{i k} \mathcal{P}^{j l}=0
$$

for $k, l=1, \ldots, m$ and $f \in \mathcal{C}^{\infty}(\Sigma)$.

Proof. One computes

$$
\begin{aligned}
{\left[\mathcal{D}^{i}, \mathcal{D}^{j}\right] } & (f) \mathcal{P}^{i k} \mathcal{P}^{j l}=\left(\mathcal{D}^{i}\left(\mathcal{D}^{j}(f)\right)-\mathcal{D}^{j}\left(\mathcal{D}^{i}(f)\right)\right) \mathcal{P}^{i k} \mathcal{P}^{j l} \\
& =-\mathcal{P}^{k}\left(\mathcal{D}^{j}(f)\right) \mathcal{P}^{j l}+\mathcal{P}^{l}\left(\mathcal{D}^{i}(f)\right) \mathcal{P}^{i k} \\
& =-\mathcal{P}^{k}\left(\mathcal{P}^{j l} \mathcal{D}^{j}(f)\right)+\mathcal{P}^{k}\left(\mathcal{P}^{j l}\right) \mathcal{D}^{j}(f)+\mathcal{P}^{l}\left(\mathcal{P}^{i k} \mathcal{D}^{i}(f)\right)-\mathcal{P}^{l}\left(\mathcal{P}^{i k}\right) \mathcal{D}^{i}(f) \\
& =\mathcal{P}^{k}\left(\mathcal{P}^{l}(f)\right)-\mathcal{P}^{l}\left(\mathcal{P}^{k}(f)\right)+\mathcal{D}^{i}(f)\left(\mathcal{P}^{k}\left(\mathcal{P}^{i l}\right)-\mathcal{P}^{l}\left(\mathcal{P}^{i k}\right)\right)
\end{aligned}
$$

by using $\mathcal{D}^{i j} \mathcal{P}^{j k}=\mathcal{P}^{i k}$ and the fact that $\mathcal{P}^{i}$ and $\mathcal{D}^{i}$ are derivations. Using the Jacobi identity in the last term yields

$$
\begin{aligned}
{\left[\mathcal{D}^{i}, \mathcal{D}^{j}\right](f) \mathcal{P}^{i k} \mathcal{P}^{j l} } & =\mathcal{P}^{k}\left(\mathcal{P}^{l}(f)\right)-\mathcal{P}^{l}\left(\mathcal{P}^{k}(f)\right)+\mathcal{D}^{i}(f) \mathcal{P}^{i}\left(\mathcal{P}^{k l}\right) \\
& =\mathcal{P}^{k}\left(\mathcal{P}^{l}(f)\right)-\mathcal{P}^{l}\left(\mathcal{P}^{k}(f)\right)+\left\{f, \mathcal{P}^{k l}\right\} \\
& =\left\{x^{k},\left\{x^{l}, f\right\}\right\}+\left\{x^{l},\left\{f, x^{k}\right\}\right\}+\left\{f,\left\{x^{k}, x^{l}\right\}\right\}=0,
\end{aligned}
$$

again by using the Jacobi identity.

It follows from the above result that $\left[\mathcal{D}^{i}, \mathcal{D}^{j}\right](f) X^{i} Y^{j}=0$ for all $X, Y \in T \Sigma$ and $f \in C^{\infty}(\Sigma)$, which implies that the Codazzi-Mainardi equations in $\left(\mathbb{R}^{m}, \delta\right)$ :

$$
X^{i} Y^{j} Z^{k}\left(\mathcal{D}^{i} \mathcal{D}^{j}\left(N^{k}\right)-\mathcal{D}^{j} \mathcal{D}^{i}\left(N^{k}\right)\right)=0,
$$

are satisfied when assuming that (3.1) holds.

\section{REFERENCES}

[AH11] J. Arnlind and G. Huisken. On the geometry of Kähler-Poisson structures. arXiv:1103.5862, 2011.

[AHH10a] J. Arnlind, J. Hoppe, and G. Huisken. Discrete curvature and the Gauss-Bonnet theorem. arXiv:1001.2223, 2010.

[AHH10b] J. Arnlind, J. Hoppe, and G. Huisken. On the classical geometry of embedded manifolds in terms of Nambu brackets. arXiv:1003.5981, 2010.

[AHH10c] J. Arnlind, J. Hoppe, and G. Huisken. On the classical geometry of embedded surfaces in terms of Poisson brackets. arXiv:1001.1604, 2010.

[AHH12] J. Arnlind, J. Hoppe, and G. Huisken. Multi-linear formulation of differential geometry and matrix regularizations. J. Diff. Geo., 91:1-39, 2012.

[Arn13] J. Arnlind. Curvature and geometric modules of noncommutative spheres and tori. arXiv:1308.3330, 2013.

[BR82] M. Barros and A. Romero. Indefinite Kähler manifolds. Math. Ann., 261(1):55-62, 1982.

[BS10] D. N. Blaschke and H. Steinacker. Curvature and Gravity Actions for Matrix Models. Class. Quant. Grav., 27:165010, 2010.

[CFG96] V. Cruceanu, P. Fortuny, and P. M. Gadea. A survey on paracomplex geometry. Rocky Mountain J. Math., 26(1):83-115, 1996.

[Cla70] C. J. S. Clarke. On the global isometric embedding of pseudo-Riemannian manifolds. Proc. Roy. Soc. London Ser. A, 314:417-428, 1970.

[Hin11] P. Hintz. The geometry of embedded pseudo-Riemannian surfaces in terms of Poisson brackets. arXiv:1107.0700, 2011. 
[Hop82] J. Hoppe. Quantum Theory of a Massless Relativistic Surface and a Two-dimensional Bound State Problem. PhD thesis, Massachusetts Institute of Technology, 1982.

[KN96a] S. Kobayashi and K. Nomizu. Foundations of differential geometry. Vol. I. Wiley Classics Library. John Wiley \& Sons Inc., New York, 1996. Reprint of the 1963 original, A Wiley-Interscience Publication.

[KN96b] S. Kobayashi and K. Nomizu. Foundations of differential geometry. Vol. II. Wiley Classics Library. John Wiley \& Sons Inc., New York, 1996. Reprint of the 1969 original, A Wiley-Interscience Publication.

[Nas56] J. Nash. The imbedding problem for Riemannian manifolds. Ann. of Math. (2), 63:2063, 1956.

(Joakim Arnlind) Dept. of Math., Linköping University, 58183 Linköping, Sweden

E-mail address: joakim.arnlind@liu.se

(Gerhard Huisken) Mathematisches Forschungsinstitut Oberwolfach, SchwarzwaldStr. 9-11, 77709 Oberwolfach-Walke, Germany

E-mail address: huisken@mfo.de 